\title{
Algorithm for calculating hazard areas of a rock massif based on geomechanical data
}

\author{
Andrei Gladyr ${ }^{*}$, Maksim Rasskazov, Alexander Konstantinov and Andrei Tereshkin \\ Mining Institute, Far Eastern Branch of the Russian Academy of Sciences, 680000, 51, Turgenev st., \\ Khabarovsk, Russian Federation
}

\begin{abstract}
Based on the analysis of existing approaches in the prediction of dynamic phenomena of rock pressure, it was established that the basis of most methods is the kinetic concept of the destruction of solids. A team of authors from the Mining Institute of the Far Eastern Branch of Russian Academy of Sciences has developed a method of geomechanical monitoring of a rock massif state, which consists of analyzing the dynamics of the acoustic active zones formation and predicting the impact hazard based on the established regularities of changes in the geoacoustic activity. This paper proposes an automated method for identifying focal zones, based on preliminary exclusion of background radiation using a non-parametric density estimation method, identifying seismoacoustic active zones by means of probabilistic cluster analysis and parameterizing focal zones by selecting a characteristic ellipsoid.
\end{abstract}

\section{Introduction}

According to the results of the analysis of existing methods for predicting the dynamic manifestations of rock pressure, it has been established that most methods working based on the kinetic concept of the destruction of solids. Many scientists who adhere to this concept have found that in the process of rock destruction several characteristic stages are distinguished. In these stages, the process of destruction begins with the formation of microcracks measuring in millimeters to centimeters and even meters, and may end with ruptures from dozens to thousands of meters. Such ruptures are characteristic of mountain tectonic impacts, man-made earthquakes, etc. [1-4].

Researchers at the Mining Institute of the Far East Branch of the Russian Academy of Sciences have developed and proposed to use a method for monitoring seismoacoustic active areas of a mountain massif $[1,6]$. The method is designed to determine the shock hazard index of a potentially dangerous focal zone, which is detected and qualified according to the results of continuous seismic-acoustic monitoring in an automated mode.

To assess the state of the rock mass, it is recommended to use the integral shock hazard indicator, which takes into account a number of signs of the rock propensity within the controlled area to dynamic manifestations.

*Corresponding author: rush3112@mail.ru 
The key point of the developed method of control the seismoacoustically active zones is the selection of a certain controlled zone, which can be qualified as a focal zone. Within this zone, a potentially dangerous source of destruction of rocks is formed. However, in these works, there is no sufficient study of a statistically based methodology for the selection of these zones, which allows to use these methods in automated systems for geomechanical monitoring. The solution to the problem of forming a characteristic volumetric figure, for example, an ellipsoid describing the geometric dimensions of the focal zone, is also not given.

\section{Materials and methods}

The practice of using cluster analysis methods for seismic acoustic monitoring data shows that before starting clustering, we should choose subsets of data located in areas with high density of points. It is proposed to estimate the distribution density at each of the points using a non-parametric density estimate [7]. Based on the model described above, the solution of this problem will allow the separation of values relating to regions with low and high density. In this case, the values assigned to the regions with low density, uniquely refer to the background radiation.

A registered seismoacoustic event with calculated coordinates $\left(x_{i}, y_{i}, z_{i}\right)$ will be considered to belong to a region of high density, if the number of points located inside a sphere centered at a point with coordinates $\left(x_{i}, y_{i}, z_{i}\right)$ and some radius $h$ is greater than a certain threshold values with.

The main task in carrying out practical calculations of the above weight function is the choice of the optimal values of the parameters $h$ and $c$. To find the parameter $h$, it is recommended to use cross-validation methods based on least squares, which minimize the mean square integral error of the calculated estimate $[8,9]$.

To automate the selection of the optimal value of $c$, the following algorithm is proposed (Fig. 1). The proposed algorithm for filtering background radiation solves the problem of removing excess data for the subsequent identification of seismoacoustic cores and allows us to classify events belonging to background radiation into a separate group. At the same time, it is possible to study the background radiation and its influence when predicting dynamic manifestations in the rock massif [10].

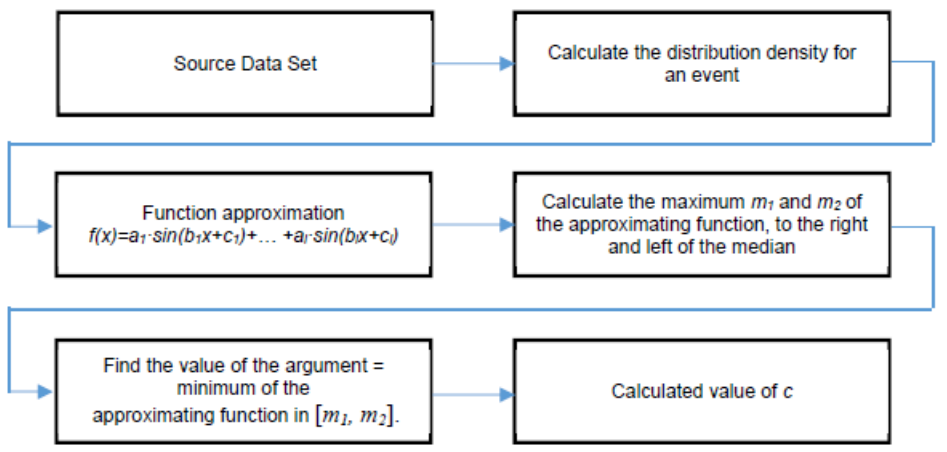

Fig. 1 Algorithm for the selection of the optimal value $c$.

Cluster analysis is the task of partitioning a given sample of objects (situations) into subsets, called clusters, so that each cluster consists of similar objects, and the objects of different clusters are significantly different [11]. 
At first view, based on the concepts of physical processes occurring in the process of loading and subsequent deformation of a mountain massif, the use of hierarchical or graph approaches seems to be most appropriate for describing focal zones [11]. Note that these approaches are built on the basis of deterministic models, since it is assumed that during the clustering process, the initial set of objects $\mathrm{X}$ is divided into several disjoint subsets. In other words, these methods are methods of "clear" clustering.

At the same time, "fuzzy" clustering methods allow the same object to belong simultaneously, but with a different degree of probability, to several (or even all) clusters. What is more natural in many situations is because it takes into account random processes characteristic of objects of nature and their stochastic nature [12].

Note that the dynamic processes occurring in the mountain massif are largely determined by a set of interrelated physical processes, which are mostly probabilistic in nature. Therefore, the use of a deterministic approach for the analysis of stochastic natural processes can be recommended solely as an initial approximation.

To solve the problem of calculating focal zones, a modified FCM algorithm - the Gustavson-Kessel algorithm is used, which divides the data into a known number of clusters described by a fuzzy partitioning matrix. At the same time, the main difference for fuzzy and clear methods is that for fuzzy partitions, the degree to which the object belongs to the cluster takes values from the interval $[0,1]$, and for clear, from the two-element set $\{0,1\}$.

In this algorithm, clustering optimizes not only the coordinates of the cluster centers and the fuzzy partition matrix, but also the norm-generating matrices for all clusters. This allows you to select clusters of various geometric shapes.

In the above algorithm, the most important parameter is the number of clusters. The optimal method for determining the number of clusters can be considered the subtractive clustering method, one of the advantages of which is the absence of the need to specify the initial number of clusters [13].

Based on the above, the following complex algorithm for the formation of focal zones based on the results of seismo-acoustic monitoring is proposed:

1. Among the total number of registered seismoacoustic events, regions with low seismoacoustic activity that are related to background radiation are filtered using a nonparametric density estimate.

2. The number of clusters is determined using the subtractive clustering algorithm.

3. The process of multiple repetition of clustering according to the Gustavson-Kessel algorithm is performed to obtain a stable result of splitting the initial set of objects into the optimal number of clusters by estimating the probability of distribution. After the process is completed, for each observation, the greatest probability that the event belongs to each cluster is calculated.

The developed algorithm allows for an additional assessment of the quality of clustering. The process of dividing objects into clusters is performed as follows: the splitting procedure is performed a large number of times, while for each event the probability of belonging to a given cluster to which it was assigned remains. Upon completion of the calculations for each event, the highest probability (in the frequency ratio) of belonging to each of the clusters is selected. Thus, the result is both the separation of events by the probability of being assigned to a particular cluster, and the accuracy of assigning each object (observation) to each of the clusters.

The location and subsequent clustering of seismo-acoustic signals make it possible to form an area of defects with the identification of the direction of propagation of cracks in space. Suppose that in space this area will have the shape of some ellipsoid with its main diagonals, which characterize the ratio of the length, width and thickness of the destroyed layer and the orientation of this layer in space. As the initial data we take the table of 
coordinates of the registered seismo-acoustic events related to each of the clusters selected at the previous steps.

To select the direction in which the coordinate distribution will be maximal, we take into account that in this case the projection of the locations of events on the direction normal will have the maximum variance and this will characterize the direction of the source length. In an orthogonal plane, we define the center thickness as a direction with minimal dispersion in the plane. Then, in the remaining third orthogonal to the two previous directions, the dispersion of the spread of points can be denoted by the width of the source.

\section{Results and discussion}

To check the quality of the developed algorithms, we analyzed the data of seismo-acoustic monitoring obtained in 2017 at the shock-hazardous Antey deposit located in Eastern Transbaikalia [14]. The parameters of seismoacoustic events registered by the automated system of rock pressure monitoring "Prognoz ADS" on one of the sections of the mine field were analyzed.

In total for 2017, 12477 seismic acoustic events were recorded. According to the results of filtering background radiation, $40.5 \%$ of events were attributed to background radiation. For the remaining events, as a result of cluster analysis, 3 clusters (active zones) were identified. Cluster number 1 with the number of related events 3662, cluster number 2 events 1633, cluster number 3 - events 2067. The results of the construction of characteristic ellipsoids describing focal regions are presented in Fig. 2 (the center of the coordinate system is brought to the center of the selected focal zone).

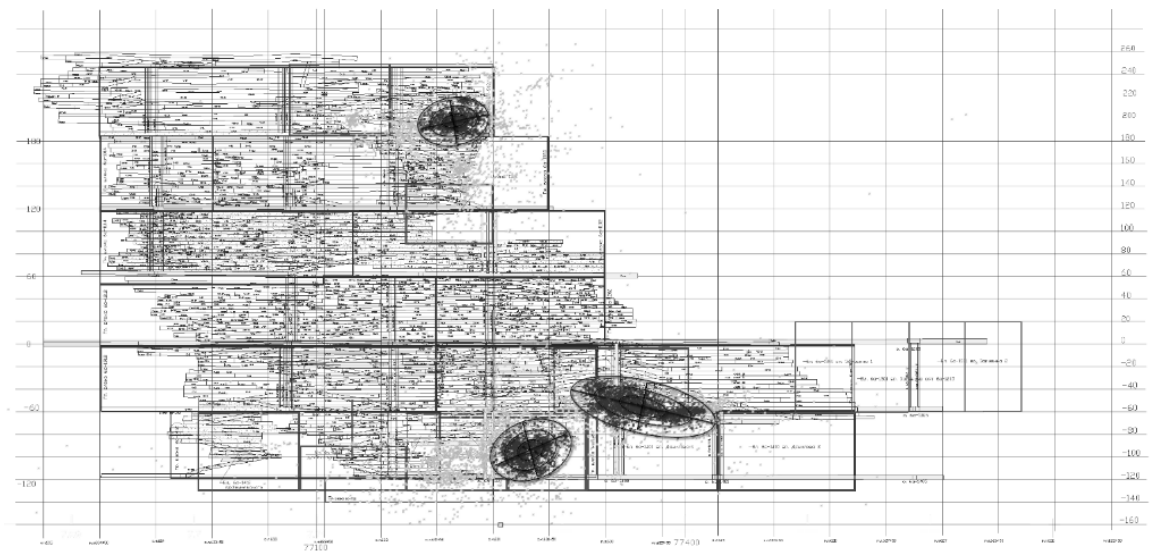

Fig. 2. The result of the work of the focal zone allocation algorithm based on seismicacoustic monitoring data. The projection on the vertical plane.

Thus, the use of the characteristic ellipsoid allowed more than 100 times reduction in the number of parameters describing the selected focal zone, while providing an opportunity to analyze changes in the volume and shape of the focal zone, both in a static representation and in dynamics.

Note that, in the overwhelming number of cases, probabilistic clustering reflects a more "natural" nature of the processes than the classical one, since it takes into account random processes characteristic of real living objects and their stochastic nature, which is particularly important for elements located on the boundary frequent cluster structures. 


\section{Conclusions}

The subsequent use of the proposed methods in geomechanical monitoring systems contributes to automating the process of predicting the dangerous state of the monitored rock massif, significantly improving the quality of the forecast, while simultaneously optimizing the temporal characteristic of obtaining the final result. This will allow the use of the developed algorithms and methods as part of the system for automated monitoring of the geomechanical state in the mine version, and as part of autonomous systems and mechanisms for open-pit mining [15-17].

\section{References}

1. I. Yu. Rasskazov, Control and management of rock pressure in the mines of the Far Eastern region (2008)

2. K. P. Zhou, Y. Lin, H. W. Deng, J. L. Li, C. J. Liu, Transactions of Nonferrous Metals Society of China, 26, 1995 (2016)

3. C. Meifeng, Journal of Rock Mechanics and Geotechnical Engineering, 8 (2), 204 (2016)

4. T. H. Ma, C. A. Tang, L. X. Tang, W. D. Zhang, L. Wang, Tunnelling and Underground Space Technology, 49, 345 (2015)

5. Shan-chao Hu, Yun-liang Tan, Jian-guo Ning, Wei-Yao Guo, Xue-sheng Liu, Shock and Vibration, 2017, 8 (2017)

6. I. Yu. Rasskazov, S. V. Tsirel, A. O. Rozanov, A. A. Tereshkin, A. V. Gladyr, Journal of Mining Science, 2, 29 (2017)

7. A. I. Orlov, In the collection: Statistical methods of evaluation and testing of hypotheses. Interuniversity collection of scientific papers, 1, 68 (1996)

8. A.W. Bowman, Biometrika, 71, 353 (1984)

9. M. Rudemo, Scandinavian Journal of Statistics, 9, 65 (1982)

10. A. V. Gladyr, Mining informative-analytical bulletin (scientific and technical journal), S21, 65 (2016)

11. V. S. Berikov, G. S. Lbov, All-Russian competitive selection of review and analytical articles in the priority area "Information and telecommunication systems", 1, 26 (2008)

12. A. D. Myshkis, Elements of the mathematical models theory (2007)

13. R. Yager, D. Filev, Essentials of Fuzzy Modeling and Control (1993)

14. I. Yu. Rasskazov, V. A. Petrov, A. V. Gladyr, D. V. Tyurin, Mining Journal, 7, 23 (2018)

15. A. Yu. Cheban, Mine surveying and subsoil use, 4, 23 (2017)

16. H. Manchao, R. Fuqiang, L. Dongqiao, International Journal of Mining Science and Technology, 28 (5), 829 (2017)

17. G. V. Sekisov, A. A. Sobolev, Mining informative-analytical bulletin (scientific and technical journal), 11, 38 (2012) 